auf einem ganz andern Gebiet der internen Medizin liegen, und der auf dem Gebiete der Tuberkulose überbaupt keine produktive Arbeit aufzuweisen hat, in der allgemeinen Urteilsbildung vielfach höher gewertet werden, als die Ergebnisse aus der mühevollen Arbeit bekannter Tuberkuloseforscher, die zufällig nicht auf die Wünde eines akademischen Titels hinweisen können.

Alles dies sind gewiß Rückständigkeiten und Organisationsfehler, die einer Verjüngung bedürfen. Diese Verjüngung darf sich aber niemals unter politischem Drucke vollziehen. Ein solcher ist auf dem Boden der Wissenschaft schärfstens abzulehnen, von welcher Seite er immer kommen mag.

Und so kann es auch niemals Sache einer Regieruing sein, in die Lösung rein wissenschaft. licher Probleme einzugreifen. Das Friedmannsche Verfahren stellt heute zwar ein sicher hochinteressantes und praktisch wichtiges - gewiß aber kein praktisch gelöstes Problem dar. Die Beurteilung einer eventuellen praktischen Verwend. barkeit in größerem Maßstabe kann daher nur der Entscheidung einer fachmännischen Prüfungskommission zustehen. Wine solche wurde bereits von der alten Regierung aufgestellt, und diese hat diesbezüglich gewiß nichts versäumt denn damals war das Friedmannsche Verfahren nicht einmal so weit, daß bei seiner Anwendung die Möglichkeit höchst unerwünschter $\mathrm{Zwischen-}$ fälle ausgeschaltet blieb.

Ohne uns durch irgendwelche persönlichen oder politischen Sympathien oder Antipathien leiten zu lassen, werden wir folgenden Wunsch aussprechen können. Friedmann soll ebenso unverdrossen wie bisher weiter arbeiten, und seiner Arbeit soll jede sachliche Förderung zuteil werden, die ihrer weiteren Entwicklung nützt. Die Politik soll aber auf diesem Gebiete schweigen und die Wissenschaft mit allzu billigen demagogischen Methoden verschonen. Dio Methode ist aber selbst für die heutige Zeit etwas zu seicht, für ein Heilverfahren dadurch Reklame zu machen, daß man hochverdiente Medizinalbeamte der Parteilichkeit beschuldigt, oder dadurch, daB man durch die Tagespresse der Bevölkerung schwungvolle Àrtikel vorsetzt, daß ein sicheres, allgemein anwendbares Heilmittel gegen die Tuberkulose gefunden worden sei, eine rückständige Regierung aber dem Volke diese segensreiche Entdeckung vorenthalten habe.

Es läBt sich heute noch nicht mit wissenschaftlichen Methoden entscheiden, ob uns die Schildkröten Friedmanns den großen Segen einer wirklich durchgreifenden, erfolgreichen Tuber kulosebekämpfung bieten können. Sicher ist aber daB sie uns auch auf dem Gebiete der Wissenschaft den Weg in einen Sumpf kulturellen Niederganges weisen, wenn es Methode werden würde, wissenschaftliche Meinungsgegensätze nicht mehr mit wissenschaftlichen, sondern mit politischen Machtmitteln auszukämpfen.

\section{Über die Reduktion der Salpetersäure in grünen Zellen.}

Fon Otto Warburg, Berlin-Dahlem 1 ).

Neben einigen Bakterien und Schimmelpilzen sind es vor allem die autotrophen Pflanzen, die ihren Stickstoffbedarf aus Nitraten decken. Der Stickstoff der Salpetersäure geht hierbei in die Reduktionsstufe des Ammoniaks über, mit der Assimilation des Stickstoffs ist also, summarisch betrachtet, folgender Reduktionsvorgang verbunden:

$\mathrm{HNO}_{3}+\mathrm{H}_{2} \mathrm{O}=\mathrm{NH}_{3}+2 \mathrm{O}_{2}-68000 \mathrm{cal}$. （1

Die Zahl -88000 berechnet sich nach H. $\left.P i c k^{2}\right)$ aus der Thermodynamik der Stickstoffverbindungen und bedeutet, daß die Zufuhr von 68000 cal. Arbeit erforderlich ist, damit der Vorgang von links nach rechts abläuft.

Näheres ist über den Verlauf dieser Reduktion nicht bekannt, wohl im wesentlichen, weil sie im normalen Stoffwechsel gegen die Oxydation der Brennstoffe völlig zurücktritt und deshalb direkt nie gemessen werden konnte. Die Nitratreduktion so zu beschleunigen, daß sie einer dírekten Messung zugänglich wurde, war die erste Aufgabe.

Als Versuchsobjekt diente eine kleine isoliert wachsende Grünalge, Chlorella vulgaris Beyerink. Bemühungen, die Reduktion hier durch Darbietung konzentrierter Nitratlösungen zu beschleunigen, waren erfolglos, dagegen fanden sich Anzeichen einer Beschleunigung in verdünnten $\mathrm{Lö}^{-}$ sungen freier Salpetersäure. $\because \mathrm{Da}$ undissozilerte Säuremoleküle, im Gegensatz zu Salzen und Ionen, vielfach schnell in lebende Zellen eindringen, lag es nahe, weitere Versuche mit höheren Konzentrationen an undissoziierter Salpetersäure anzustellen. Macht man eine 1/100 normale Salpetersäurelösung in bezug auf Nitrat $1 / 10$ normal, so steigt die Konzentration des undissoziierten Säureanteids auf etwa das Neunfache. In einem derartigen Säurenitratgemisch verläuft die $R e$ duktion der Salpetersäure so schnell, daß sie in Dunkeln $50 \%$, bei Bestrahlung $150 \%$ des Gesamtstoffwechsels ausmacht, in beiden Fällen also obne Schwierigkeiten direkt gemessen werden kann. Eine Schädigung der Algen tritt hierbei innerhalb der Versuchszeiten nicht ein, die Zellen bleiben unverändert grün und teilungsfähig.

Die zunächst zu beschreibenden Versuche sind Dunkelversuche. Werden die Algen in ihrer $\mathrm{Kul}$ turflüssigkeit, der Knopschen Lösung, gehalten, so erscheint im Dunkeln für ein Volumen veratmeten Sauerstoffs ein Volumen Kohlensäure; die Gleichung der Atmung lautet also:

$$
\mathrm{O}_{2}+\mathrm{C}=\mathrm{CO}_{2}+115000 \mathrm{cal} \text {. . . . . (2 }
$$

worin +118000 cal. in abgerundeter Zahl die

1) Nach einem vor der deutsehen Physiologentagung in Hamburg am 28. Mai gehaltenen Vortrag.

2) H. Pick, Zeitschrift f. Elektrochem. Bd. 26 , S. 182 (1920). Uber die Bedingungen der Konzentrationen und Drucke vgl. die in der Bioch. Zeitschrift erscheinende ausfuhrliche Arbeit. 
Änderung der freien Energie bei Verbrennung von $1 / 6-\mathrm{Mol}$-Glucose bedeutet.

Bringen wir die Alge aus der Knopschen Lösung in das Salpetersäure-Nitratgemisch, so steigt die . Kohlensäureproduktion sofort sehr bedeutend an, auf ein Volumen veratmeten Sauerstoffs wird nicht ..ein...Volumen: Kohlensäure ausgeschieden, sondern etwa $50 \%$ mehr. Neben der Atmungskohlensäure erscheint also in dem Nitratgemisch Kohlensäure anderer Herkunft, die wir im folgenden Extrakohlensäure nennen wollen.

Es läßt sich zeigen, daß die Ausscheidung der Extrakohlensäure mit einer Reduktion dor Salpetersäure in Zusammenhang steht, indem gleichzeitig mit der Extrakohlensäure ein Reduktionsprodukt der Salpetersäure, Ammoniak, an die umgebende Flüssigkeit abgegeben wird. LäBt: man der Alge zunächst einige Stunden Zeit, ihren Stickstoffbedarf zu decken und miBt dann gleichzeitig die Ausscheidung von Extrakohlensäure und Ammoniak, so findet man, daß mit jedem Molekül Ammoniak 2 Moleküle Extrakohlensäure erscheinen, entsprechend ifolgender Gleichung :

$\mathrm{HNO}_{3}+\mathrm{H}_{2} \mathrm{O}+2 \mathrm{C}=\mathrm{NH}_{3}+2 \mathrm{CO}_{2}+162000$ cal. (3

Wie also bei der Explosion des Schießpulvers Salpeter und Kohle unter Bildung von Stickstoff und Kohlenoxyd reagieren, so geht in der lebenden Zelle ein innerer Verbrennungsprozeß vor sich, der zu den Endprodukten Ammoniak und Kohlensäure führt. Diese Reaktion bedarf keiner Zufuhr von Arbeit, sondern verläuft mit einem Arbeitsgewinn von 162000 cal.

Reagiert hier der Nitratsauerstoff mit dem Kohlenstoff organischer Verbindungen ähnlich wie der freie Sauerstoff in der Atmung, so liegen beiden Vorgängen doch durchaus verschiedene Mechanismen zugrunde, wie folgender Versuch zeigt: Die Sauerstoffatmung der Alge ist wenig empfindlich gegenüber Blausäure. Um den Verbrauch von Sauerstoff und die Bildung von Atmungskohlensäure merklich zu verlangsamen, muß die Blausäurekonzentration den hohen Wert von $1 / 10$ Mol pro Liter erreichen. Im Gegensatz hierzu wird die Reduktion der Salpetersäure zu Ammoniak schon durch kleine Blausäuremengen völlig gehemmt. Setzen wir einem Liter Nitratgemisch ein hunderttausendstel Mol Blausäure zu, so scheiden die Algen weder Extrakohlensäure noch Ammoniak aus, und wir haben Zellen, die zwar unverändert atmen, jedoch nicht mehr imstande sind, Salpetersäure zu Ammoniak zu reduzieren. Die Salpetersäure reagiert also mit dem Kohlenstoff der Zelle in einem Mechanismus sui generis; der sich von dem Atmungsmechanismus durch die zehntausendfache : Empfindlichkeit gegenüber Blausäure unterscheidet.

Die Wirkung der Blausäure auf Lebensvorgänge beruht aller Wahrscheinlichkeit auf ihrer Fähigkeit, ISchwermetalle aus einer katalytisch wirksamen Form in katalytisch unwirksame kom- plexe Cyanide überzuführen. Auch bei der Nitratreduktion scheinen also, wie bei so vielen andern Lebensvorgängen, Schwermetallspuren eine Rolle zu spielen.

Die bisher beschriebenen Versuche waren mit Nitratgemischen angestellt, deren Konzentration an freiem Sauerstoff durch dauerndes Schütteln mit atmosphärischer Luft auf einer bestimmten konstanten Höhe gehalten war. Lassen wir jedoch den Sauerstoffgehalt sinken, etwa indem wir in luftdicht verschlossenen Gefäßen arbeiten, so ist die Alge nicht mehr imstande, Salpetersäure zu Ammoniak zu reduzieren. Bei niedrigen Konzentrationen an freiem Sauerstoff erscheint ein anderes $\mathrm{Re}$ duktionsprodukt der Salpetersäure, die salpetrige Säure. 'Diese zweite Art von Reduktion, die offenbar mit der in der Natur wichtigen Reduktion zu Ammoniak nichts gemein hat, verläuft bei SauerstoffabschluB so lebhaft, daß die Alge innerhalb weniger Stunden an Nitritvergiftung zugrundegeht, wobei die grüne Farbe des Chlorophylls in braun umschlägt.

Soviel über die Versuche im Dunkeln. Was den Einfluß der Bestrahlung auf die Nitratreduktion anbetrifft, so sind zunächst einige frühere Arbeiten zu erwähnen. Schimper ${ }^{1}$ ). verfolgte mittels der Diphenylaminprobe das Schicksal der Nitrate in grünen Blättern und beobachtete, daB der Nitratgehalt nur bei Bestrahlung merklich abnahm. Godlewski²) stellte Stickstoffbilanzen für dunkel und hell gezogene Weizenkeimlinge auf und kam zu dem Schluß, daß ,das Licht auf die Verarbeitung der Nitrate - begünstigend einwirkt". Godlewskis Auffassung gilt heute allgemein als richtig, geteilt sind die Ansichten nur über die Art und Weise, wie dieser begünstigende Einfluß des Lichts zustandekommt. Hierüber geben, wie ich glaube, die Bestrahlungsversuche mit Chlorella nähere Aufschlüsse.

Bestrahlen wir die Alge in dem Nitratgemisch, so erscheint mehr Ammoniak und gleichzeitig etwa dreimal soviel Extragas, als im Dunkeln. Dieses Extragas ist jedoch nicht, wio in den Dunkelversuchen, Kohlensäure, sondern Sauerstoff. Summarisch formuliert, entspricht der Vorgang Gleichung 1, indem die erforderlichen $68000 \mathrm{cal}$. Arbeit durch die absorbierte Strahlung geleistet werden.

Aus der Tatsache, daB im Dunkeln Ammoniak und Kohlensäure, bei Bestrahlung Ammoniak und Sauerstoff als Endprodukte auftreten, darf nicht geschlossen werden, daß der Verlauf der Nitratreduktion in beiden Fällen ein verschiedener ist. Im Gegenteil war von vornherein wahrscheinlich, da $B$ die Salpetersäure in beiden Fällen in demselben; durch Bestrahlung nur beschleunigten $\mathrm{Me}$ chanismus reduziert werde; bei Bestrahlung käme

1) $F$. Wh Schimper, Bot. Zeit. Bd. 46, S. 65 (1888).

2) E. Godlewski, Extrait Bull. Acad. Scienc. Cracovie, Juin 1903. 
dann als zweiter, von der Nitratreduktion unabhängiger Vorgang die Kohlensäureassimilation hinzu und würde die bei der Nitratreduktion entstehende Kohlensäure in Sauerstoff und Kohlenstoff zurückverwandeln. Aus der Bilanz fällt so die Kohlensäure heraus, wie sich durch Addition der Gleichungen der inneren Verbrennung (3) und der Kohlensäureassimilation (4) ergibt: $\mathrm{HNO}_{3}+\mathrm{H}_{2} \mathrm{O}+2 \mathrm{C}=\mathrm{NH}_{3}+2 \mathrm{CO}_{2}+162000 \mathrm{cal}$. (3 $2 \mathrm{CO}_{2}=2 \mathrm{C}+2 \mathrm{O}_{2}-230000 \mathrm{cal}$. (4

$\mathrm{HNO}_{3}+\mathrm{H}_{2} \mathrm{O}=\mathrm{NH}_{3}+2 \mathrm{O}_{2}-68000$ cal. (1

Durch einen einfachen Versuch läßt sich zeigen, daß diese Auffassung richtig ist. Die Kohlensäureassimilation ist besonders empfindlich gegen Narkotika, schon sehr geringe Mengen dieser Stoffe heben, die Fähigkeit der Zelle, Kohlensäure photochemisch zu reduzieren, völlig auf. Im Gegensatz hierzu ist die innere Verbrennung nach Gleichung 3 gegen Narkotika unempfindlich. Wir können uns also Zellen. verschaffen, in denen der Vorgang nach Gleichung 3. wenig beeinfluBt, der Vorgang nach Gleichung 4 völlig gehemmt ist. Bestrahlen wir derartige Zellen, so muB, wenn unsere Auffassung richtig ist, mehr Kohlensäure erscheinen, als im Dunkeln. In der Tat steigt die Ausscheidung an Extrakohlensäure auf das Dreifache, wenn wir narkotisierte Zellen bestrablen.

Wir haben also in grünen Zellen zwei durchaus verschiedene Wirkungen der Bestrahlung zu unterscheiden, neben der Kohlensäureassimilation, in der die absorbierte Strahlung Arbeit leistet, die Beschleunigung eines von selbst verlaufenden Vorgangs. Beiden Wirkungen scheint derselbe photochemische Primärvorgang zugrunde zu liegen, da Strahlen vérschiedener Wellenlänge die Nitratreduktion nach Maßgabe ihrer Absorption durch das Chlorophyll beschleunigen.

\section{Die organische Elementarsynthese in der Technik.}

Von Erich Baum, Solln b. München. (Fortsetzung.)

Ein altes Problem der organischen Technik ist die Darstellung von „Mineralspiritus“, d. h. die Darstellung von Athylalkohol durch Synthese. Bereits um die Mitte des vorigen Jahrhunderts dachte man daran, Athylen, das als Nebenprodukt der Mineralölfabrikation leicht zugänglich schien, mittels Schwefelsäure in Äthylschwefelsäure zu überführen und diese dann in Schwefelsäure und Athylalkohol zu spalten ${ }^{1}$ ). Die Durchführung des Verfahrens scheiterte an dem großen Schwefelsäureverbrauch"): Utbrigens ist seine Anwendung auf Äthylen aus Kokereigas neuerdings in England wieder vorgeschlagen worden ${ }^{3}$ ). 245.

1) Wagners Jahr. Ber. d. Chem. Techn. 1856, 2,

2) Chem. Ind. 20266 (1897)

3) Ztschr. f. Carbid 11. Acetylen 1919, 72.
Die technische Lösung dieser Aufgabe, die Alkoholsynthese', ist im Gefolge einer Reihe anderer wichtiger Synthesen in den letzten Jahren vom Acetylen aus - über den Acetaldehyd gelungen.

Kutscherow ${ }^{1}$ ) hatte bei der Einwirkung von Acetylen auf Quecksilberchloridlösung die Bildung eines weißen Niederschlags beobachtet, der beim Erwärmen mit Salzsäure Acetaldehyd liefert. Es findet, wenn man die Reaktion unter Vernachlässigung der Rolle betrachtet, die dabei die Quecksilberverbindung, ein Trichlormercuriacetaldehyd2) spielt, eine Anlagerung von Wasser an Acetylen unter Bildung von Acetaldehyd statt:

$$
\mathrm{C}_{2} \mathrm{H}_{2}+\mathrm{H}_{2} \mathrm{O}=\mathrm{CH}_{3} \mathrm{CHO} \text {. }
$$

Schon 1895 wurde vorgeschlagen, durch Reduktion (mittels Zink und Säuren) aus dem so erhaltenen Aldehyd Alkohol zu gewinnen ${ }^{3}$ ); eine praktische Durchführung dieses Vorschlages war natürlich ausgeschlossen.

Später wurde beobachtet, daB auch Schwefelsäure die Bildung von Acetaldehyd aus Acetylen und Wasser in geringem Umfang bewirkt); die Reaktion tritt in Spuren übrigens bereits beim Erhitzen von Acetylen und Wasser mit Holzkohle auf $300^{\circ}$ ein $)$. Erdmann und Köthner ver suchten die Wirkung von Quecksilbersalz und Schwefelsäure zu vereinigen, indem sie Acetylen durch kochende wässerige Schwefelsäure unter Zusatz von Quecksilbersulfat ${ }^{6}$ ) leiteten. $\mathrm{Ob}$ gleich dabei die Ausbeute an Acetaldehyd nur sehr gering war, hatte Erdmann bereits in einem Festvortrag ${ }^{7}$ ), der das Gebiet der Acetylenverwertung für damalige Verhältnisse allzu optimistisch darstellte, die kontinuierliche Herstellung des „Mineralspiritus" auf diesem Wege in Aussicht gestellt.

A. Wunderlich hat dann gefunden ${ }^{8}$ ), daß beim Arbeiten mit schwefelsauren Quecksilbersalzlösungen unterhalb der Siedetemperatur Acetaldehyd in guter Ausbeute erhalten wird ${ }^{9}$ ).

Unabhängig von Wunderlich hat später $N$. Grünstein ${ }^{10}$ ) sich dem Studium der Reaktion ein. gehend gewidmet und ist zu einer ähnlichen $\mathrm{Ar}$ beitsweise gelangt $\left.{ }^{11}\right)$. Er hat seine Erfahrungen in einer Reihe von Patenten niedergelegt12). Grünstein arbeitet mit Lösungen von Quecksilbersalz und Schwefelsäure bei niederen Temperaturen,

1) Ber, d. Deutsch. Chem. Ges. 17, 13.

2) Biltz und Humm, Ber. d. Deutsch. Chem. Ges. 3\%, 4417

3) Chem. Ind. 1895,458 .

1) Ann. de Chim de Phys. (6) 15, 268; Ber. d. Deutsch. Chem. Ges. 10, 637.

5) Bull. de la Soc. Chim. 11362.

o) Ztschr. f. anorg. Chem. 18,55.

7) Chem. Ztg. $1898,869$.

8) Pat. Anm. W. $27177 \mathrm{Kl} .120$ vom 9. 2. 1907 Friedländer, Fortschritte der Teerfarbenfabrikation, $I X, 55$.

9) Ztschr. f. ang. Chem. 1918, 180; 1919, 132 ,

10) N. Grünstein, Ztschr, f. angew. Chem. 1919, 104.

11) Ztschr. f. angew. Chem. 1919, 32 .

12) Ztschr. f. angew, Chem. 1919, 104. 